\title{
Microorganism: natural sweepers of arsenic in industrial wastewater
}

\author{
A.B. Kashif Hayat ${ }^{1,2}$, C.D. Saiqa Menhas ${ }^{2}$, J. Bundschuh ${ }^{3}$, P. Zhou ${ }^{1}$ \& H.J. Chaudhary ${ }^{2}$ \\ ${ }^{1}$ School of Agriculture and Biology, Key Laboratory of Urban Agriculture, Ministry of Agriculture, \\ Bor S. Luh Food Safety Research Center, Shanghai Jiao Tong University, Shanghai, China \\ ${ }^{2}$ Department of Plant Sciences, Faculty of Biological Sciences, Quaid-i-Azam University, Islamabad, Pakistan \\ ${ }^{3}$ UNESCO Chair on Groundwater Arsenic within the 2030 Agenda for Sustainable Development, \\ University of Southern Queensland, Toowoomba, QLD, Australia
}

\begin{abstract}
The presence of arsenic (As), a potent carcinogen, in industrial wastewater poses a serious threat to plant, animal, and public health, especially in the transition and developing countries. Recent developments in the field of bioremediation have enhanced our understanding about the microbial biotechnology processes governing As uptake and detoxification on laboratory and pilot scale. In particular, the biofilm and consortia remediation will prove invaluable in the developments of new strategies to mitigate this threat at industrial scale also. The role of microbial remediation of As in industrial wastewater cannot be neglected due to its proven significance as a natural sweeper that can mitigate As in wastewater without production of solid/liquid waste and reduce it to low toxic level.
\end{abstract}

\section{INTRODUCTION}

One of the most burning issues confronting the world is the administration and sustainable management of a silent killer and carcinogen, i.e. arsenic (As) in industrial wastewater. In fact, many conventional techniques being applied to treat As in industrial wastewater face limitations in the form of missing technical expertise, low effectiveness, high costs, and production of toxic waste (Bundschuh and Maity, 2015). In this regard, microbial biotechnology emerged as an effective process for As mitigation in industrial wastewater (Hayat et al., 2017).

\section{MICROBES: AN INTENGIBLE SWEEPERS OF ARSENIC IN INDUSTRIAL WASTEWATER}

Microorganisms may be termed as an intangible and invisible natural sweeper of As in industrial wastewater. A diverse group of microorganisms plays a key role in the fate of As the biochemical cycle in industrial wastewater (see Fig. 1). Microorganisms have developed a lot of mechanisms to decrease the adverse effects of As in industrial wastewater, thus leading to positive effects on environment and human health. The key benefit of these microbes is that it may not produce any waste and cope with the As-rich environment effectively (Hayat et al., 2017). These microbes may be used individually or as a contingent termed as "biofilm or consortia". The application of algae and biofilm biotechnology is turning to be the

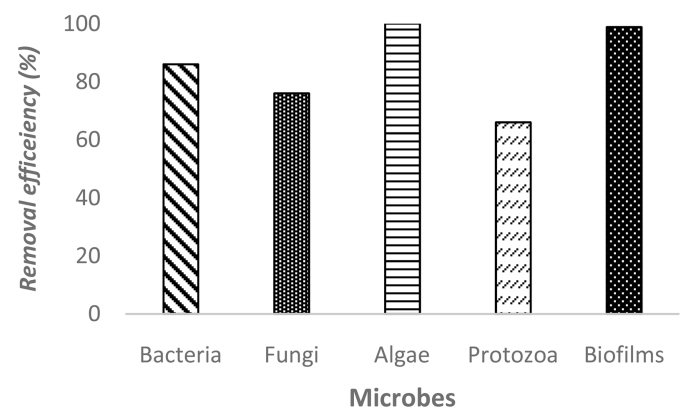

Figure 1. Removal efficiency (\%) of arsenic by microbes in industrial wastewater (Hayat et al., 2017).

most effective approach to treat As (98.8 to $100 \%)$ in industrial wastewater. Beside all other microbial technologies, biofilm (600 times more resilient than individual microbes) has been successfully used on a pilot scale due to its long-lasting effect (BattagliaBrunet et al., 2006; Dastidar and Wang, 2009; Hayat et al., 2017).

\section{CONCLUDING REMARKS AND FUTURE PROSPECTS}

In the last decade, the scientific community focused on the microbial removal of As from industrial wastewater and it was revealed that all the microbes follow a common ideal mechanism of As mitigation by which they can remove As releasing it into the atmosphere without producing solid/liquid waste. Thus, the treated 
wastewater can be regarded as within the safe limit by the environmentalist and other global scientific community. Moreover, following the recent successful trend of using natural as well as genetically engineered (GE) microbes (without avoiding the GE conflict) for As removal from industrial wastewater, there are also some other options such as nanotechnology and DNA editing technology (CRISPR) and other new molecular mechanism that should be applied and tested to reduce As concentration in industrial wastewater. However, microbial biotechnology has not yet been applied on an industrial scale (and only biofilms on pilot scale). Therefore, more insights into the microbial arsenic mitigation in industrial wastewater are urgently required.

\section{REFERENCES}

Battaglia-Brunet, F., Joulian, C., Garrido, F., Dictor, M.-C., Morin, D., Coupland, K., Johnson, D.B., Hallberg, K.B. \& Baranger, P. 2006. Oxidation of arsenite by Thiomonas strains and characterization of Thiomonas arsenivorans sp. nov. Antonie van Leeuwenhoek. 89(1): 99-108.

Bundschuh, J. \& Maity, J.P. 2015. Geothermal arsenic: occurrence, mobility and environmental implications. Renew. Sust. Energ. Rev 42, 1214-1222.

Dastidar, A. \& Wang, Y.T. 2009. Arsenite oxidation by batch cultures of Thiomonas arsenivorans strain b6. J. Environ. Eng. 135(8): 708-715.

Hayat, K., Menhas, S., Bundschuh, J. \& Chaudhary, H.J. 2017. Microbial biotechnology as an emerging industrial wastewater treatment process for arsenic mitigation: a critical review. J. Clean. Prod, 151: 427-438. 